\title{
Selected papers of the 15th International Conference of the International Society for Scientometrics and Informetrics (ISSI), Boğaziçi University, Istanbul, Turkey, 29 June-4 July 2015
}

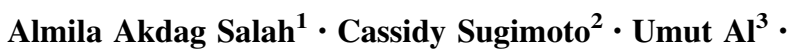 \\ Wolfgang Glänzel ${ }^{4}$
}

Received: 18 March 2016/Published online: 5 April 2016

(C) Akadémiai Kiadó, Budapest, Hungary 2016

The 15th International Society of Scientometrics and Informetrics Conference took place at Boğaziçi University in Istanbul, from June 29 to July 4, 2015. The Conference was jointly organised by Boğaziçi University, Hacettepe University, and the Turkish Academic Network and Information Center of the Scientific and Technological Research Council of Turkey (TÜBITAK ULAKBIM) under the auspices of ISSI-the International Society for Scientometrics and Informetrics.

The ISSI biennial conference series began in Belgium in 1987, and has been held biennially ever since. ISSI has been organized in five different continents and in 14 different countries: Canada (1989), India (1991), Germany (1993), USA (1995), Israel (1997), Mexico (1999), Australia (2001), China (2003), Sweden (2005), Spain (2007), South Africa (2009), Austria (2013), and Turkey (2015). As such, ISSI represents a long tradition in the scientometrics community that brings together scientists, research managers, administrators, and information professionals from diverse geographic locations.

The conference series' focus has widened over the years from theoretical and quantitative discussions to examinations of practical, cross-cultural, and multi-disciplinary aspects of information and library science, R\&D management, as well as science policy and ethics. ISSI 2015 stressed the high diffusion of scientometric and informetric research

Organised jointly by Boğaziçi University, Hacettepe University and the Turkish Academic Network and Information Center (ULAKBIM) under the auspices of the International Society for Scientometrics and Informetrics (ISSI).

Almila Akdag Salah

alelma@gmail.com

1 E-Humanities Group, KNAW, Amsterdam, Netherlands

2 School of Informatics and Computing, Indiana University Bloomington, Bloomington, IN, USA

3 Department of Information Management, Hacettepe University, Ankara, Turkey

4 ECOOM and Department of MSI, KU Leuven, Louvain, Belgium 
into the everyday life of science policy makers and scientists, inviting its participants to talk about "the future of scientometrics". Scientometrics has been responsible for creating tools for research assessment and evaluation, as well as for use in charting the flow of scientific ideas and people. These advances raised critical concerns on issues such as the inappropriate use of indicators and the meaning of and interpretation of rankings. The main question facing the field is how best to move forward given the computational opportunities and the sociological concerns.

The diversity and number of papers submitted to ISSI15 showed that the community embraced these concerns, and is ready to move forward. From 228 full and research-inprogress paper submissions, 82 full papers and 41 research-in-progress papers were accepted. The foci of paper submissions revealed community interest in social media, technology transfer, science policy, patent analysis, and research assessment. From 123 poster and ignite talk submissions, 68 posters and 13 ignite talks were accepted. In total, 458 researchers spanning 42 countries were represented as authors of these scholarly works.

For this special issue, we invited authors of full and work-in-progress papers to submit extended versions of their research, based on the feedback they have received from the peer-review process, as well as from the discussions held during the conference. Out of these submissions, which went through a second round of intense peer review, we selected 31 original research papers. This collection reflects the wide coverage of the conferencespanning novel topics such as altmetrics, webometrics, patent analysis, and contemporary science policy concerns, without neglecting core scientometric research areas such as citation analysis, indicators, visualization and mapping techniques, as well as methodological concerns.

The next event of this conference series, the 16th International Conference of the International Society for Scientometrics and Informetrics, will be held in Wuhan, China, in 2017. 